\title{
Téoros
}

Revue de recherche en tourisme

\section{Le consommateur culturel comme segment de marché de l'offre touristique}

\section{François Colbert et Jacques Boisvert}

Volume 7, numéro 1, mars 1988

Cultures régionales et tourisme

URI : https://id.erudit.org/iderudit/1080427ar

DOI : https://doi.org/10.7202/1080427ar

Aller au sommaire du numéro

Éditeur(s)

Université du Québec à Montréal

ISSN

0712-8657 (imprimé)

1923-2705 (numérique)

Découvrir la revue

Citer cet article

Colbert, F. \& Boisvert, J. (1988). Le consommateur culturel comme segment de marché de l'offre touristique. Téoros, 7(1), 17-20.

https://doi.org/10.7202/1080427ar d'utilisation que vous pouvez consulter en ligne.

https://apropos.erudit.org/fr/usagers/politique-dutilisation/ 


\section{Le consommateur culturel comme segment de marché de l'offre touristique}

L'une des tâches principaies du marketing consiste à diviser le marché global en sousgroupes de consommateurs (segments) possédant des caractéristiques identiques et qui donc seront susceptibles de réagir positivement et de la même façon à des stratégies spécifiques de mise en marché. En ciblant ses efforts vers des catégories précises de clients, l'entreprise cherche à maximiser son efficacité en répondant mieux que ses concurrents aux besoins de ce segment de marché. La segmentation des marchés est un outil de marketing rendu indispensable par la proliferation des produits. Les domaines du loisir et de la culture n'échappent pas à cette réalité.

En effet, les années soixante et soixante-dix ont vu croître de façon considérable la demande en loisir; les Canadiens dépensaient $\$ 920$ millions en 1964 pour acheter des billets de spectacles, des livres ou pour assister à des rencontres sportives alors que leur consommation s'est accrue pour atteindre en 1984, $\$ 5.3$ milliards $^{(1)}$ en dollars constants de 1964. Dans la même période de temps cependant, l'offre en loisir a subi une croissance importante, probablement supérieure en fait au taux de croissance de la demande.

Cette évolution spectaculaire nous amène cependant à une fragmentation à outrance du marchécit?,$c^{\prime}$ est-à-dire à une situation ou chaque concurrent doit se démarquer pour survivre dans un environnement hautement compétitif. Les produits de loisirs qui voient le jour sont alors ciblés de façon de plus en plus précise et visent donc une clientèle spécifique.

Le domaine du tourisme connait le même phénomène. Chaque région du Québec cherche à mettre en valeur aux yeux du public, qui ses paysages ou beautés naturelles, qui ses attraits culturels, par des manifestations populaires (Festival du bleuet), ou par des événements artistiques (Musées ou Festivals de théâtre ou de musique). L'industrie touristique fait partie intégrante du domaine du loisir et de ce fait subit la fragmentation du marché que nous y connaissons. En ce sens, pour une région définie, le consommateur culturel qui réside à l'extérieur de cette région peut représenter un segment de marché intéressant et il est possible d'ajuster ses stratégies de mise en marché de façon à attirer cette clientèle touristique particulière.

\section{Le consommateur culturel}

Mais d'abord, examinons ce consommateur culturel, qui il est, quelles sont ses caracté- ristiques et ses preférences.

On peut entendre consommateur culturel dans un sens très large ou au contraire plus restreint. Pour le moment, nous examinerons le profil socio-démographique de la clientèle qui assiste aux spectacles de thếtre, danse, musique classique ou opéra. Nous verrons ensuite comment extrapoler les connaissances que nous avons de ce spectateur particulier pour définir le consommateur de l'art que l'on pourrait qualifier de populaire.

L'ensemble de études réalisées à travers les pays industrialisés démontre que le consommateur qui achète un billet pour une compagnie en art d'interprétation se distingue du reste de la population par son revenu et son niveau de scolarisation en moyenne beaucoup plus élevés que le reste de la population. Ainsi, alors que la proportion de citoyens détenant un diplôme universitaire atteint à peine $10 \%$ au Québec, on en trouve entre 40 et $70 \%$ dans les auditoires des compagnies d'art d'interprétation. Le Conseil des arts du Canada a publié un résumé d'un certain nombre d'enquêtes effectuées au Canada et ailleurs dans le monde ${ }^{(3)}$; il semble que le niveau de scolarisation du public des arts de la scène soit le même aussi bien au Canada, aux États-Unis, en France, en Suède ou en Australie.

Somme toute, si la moitie ou les deux tiers de l'auditoire de ces compagnies est formé de gens fortement scolarisés, nous pouvons affirmer que l'une des caractéristiques de ce segment de marché est liée à cette variable. Même si le niveau de scolarisation d'un individu s'avère un bon prédicteur de son goût pour les choses artistiques, force est de constater cependant qu'il ne s'agit pas d'une donnée qui explique totalement le comportement de cette partie de la population. En effet, il existe quand mème parmi ces auditoires une bonne proportion de spectateurs qui n'ont pas dépassé le diplôme de niveau secondaire; dans la mêtme ligne de pensée, on trouvę chez les personnes fortement scolarisées un bon nombre de gens qui ne manifeste aucun goût pour la chose artistique. La variable scolarisation explique done une partie mais pas touté là réalité.

On peut par ailleurs apporter deux précisions à la description de ce consommateur culturel. D'abord, d'après l'étude de Book et Globerman ${ }^{(4)}$, on s'aperçoit que la scolarisation et les revenus augmentent avec la fréquence de consommation. Ainsi, dans I'auditoire formé des consommateurs qui déclarent aller 
TABLEAU 1

Quatre variables pour quatre événements

\begin{tabular}{|c|c|c|c|c|}
\hline & $\begin{array}{l}\text { \% avec diplôme } \\
\text { universitaire }\end{array}$ & $\begin{array}{l}\text { Fréquence de } \\
\text { visites à un } \\
\text { événement } \\
\text { culturel(souvent, } \\
\text { très souvent) }\end{array}$ & $\begin{array}{l}\text { \% hors } \\
\text { Québec }\end{array}$ & $\begin{array}{l}\text { \% événement } \\
\text { comme principale } \\
\text { raison du } \\
\text { séjour }\end{array}$ \\
\hline $\begin{array}{l}\text { 1. Théátre des } \\
\text { Amériques }\end{array}$ & $67 \%(12)^{*}$ & $89 \%$ & $11 \%$ & $46 \%$ \\
\hline $\begin{array}{l}\text { 2. Exposition } \\
\text { Picasso }\end{array}$ & $66 \%(16)$ & $62 \%$ & $36 \%$ & $22 \%$ \\
\hline $\begin{array}{l}\text { 3. Festival Juste } \\
\text { pour Rire } \\
\text { 4. Grand Prix } \\
\text { Labatt }\end{array}$ & $42 \%(29)$ & $47 \%$ & $7 \%$ & $27 \%$ \\
\hline
\end{tabular}

* \% avec diplome primaire-secondaire.

$* * \mathrm{~N} / \mathrm{O}=$ non disponible.

quatre fois ou plus au thêttre dans l'année, $48 \%$ détiennent au moins un premier cycle universitaire par rapport à $37 \%$ pour ceux qui ne déclarent y aller qu 'une fois. Dans ce segment du marché culturel, on peut déjà voir qu'il existe des sous-groupes distincts. Dans la méme étude. on faisait une distinction entre les spectateurs des grands et des petits théătres à Toronto. Encore une fois, on constate certaines différences dans le niveau de scolarisation; les petits théâtres présentent, règle générale, des oeuvres plus récentes et plus d'avant-garde et ils attirent un public encore une fois plus scolarisé. Cette donnée trouve un écho dans l'enquête effectuée par Demand Research Inc. ${ }^{(5)}$ sur le public de la danse classique et celui de la danse moderne. On y constate un niveau de scolarisation légèrement plus fort chez les spectateurs de la deuxième catégorie par rapport à ceux de la première $(50 \%$ vs $45 \%$ de diplômés universitaires).

En définitive, il semble donc exister des extrêmes parmi les consommateurs des arts d'interprétation. Le niveau de scolarisation du public varie selon la fréquence d'achat et le type de produits offerts. Certaines formes d'art exigent un public que l'on pourrait qualifier de plus averti alors que d'autres attirent un public plus large (plus élitiste versus plus populaire).

Nous avons traité jusqu'ici du secteur des arts d'interprétation. Soulignons que la meme situation existe du côté des musées et galeries d"art. Une étude américaine ${ }^{(6)}$ révèle, par exemple, qu'aux États-Unis la clientèle des musées d'art détient au moins un diplôme collégial dans une proportion variant de 41 a $66 \%$ selon I'institution, alors que pour les autres types de musées (science, histoire, anthropologie, ....) cette proportion varie de 10 à $53 \%$ (ce pourcentage est de $13.9 \%$ pour la population en général, âgée de 24 ans et plus).

\section{Le consommateur culturel touristique}

Contrairement à la situation que l'on retrouve aux États-Unis, où un bon nombre d'auteurs se sont penchés sur la question ${ }^{(7)}$, peu d'études ont été réalisées sur le consommateur culturel touristique. Par contre, à la lumière de certaines enquêttes récentes sur la clientèle de grands événements à Montréal, nous pouvons tirer un certain nombre de conclusions intéressantes. Ainsi, Éconosult Inc.(8) produisait pour le compte de quatre organismes gouvernementaux les résultats d'une collecte de données auprès du public de huit événements de Rendez-vous Montréal 1985. En conservant toujours notre critère de scolarisation comme mesure du segment de marché des amateurs d'art, nous constatons des différences significatives selon l'événement consideré. Nous avons choisi de reproduire au tableau 1 les résultats de quatre variables pour quatre événements différents; ces événements sont le Festival de théâtre des Amériques, l'Exposition Picasso, le Festival Juste pour Rire, le Grand Prix Labatt. Les variables retenues sont le pourcentage du public détenant au moins un diplôme universitaire, la fréquence de visites à d'autres manifestations culturelles, la proportion du public originaire de l'extérieur du Québec, et le pourcentage de cette dernière catégorie de public qui ont donné l'événement comme principale raison du séjour.

L'observation de la colonne "diplôme universitaire" nous permet de constater que la proportion de détenteurs d'un premier cycle est deux fois plus élevée pour un événement que l'on pourrait qualifier de plus "élitiste" ques), par rapport à un événement plus "populaire" ** (Grand Prix Labatt), alors que les détenteurs d'un diplôme d'études des niveaux primaires ou secondaires sont trois fois plus nombreux dans le deuxième cas que dans le premier. Nous voyons aussi que le Festival Juste pour Rire, se rapproche plus de la manifestation populaire que ce n'est le cas pour les deux premiers. Ces informations nous permettent donc de compléter ce que nous savons du consommateur culturel et de poser l'hypothèse que le marchế touristique peut être représenté par un continuum basé sur la variable scolarité où les segments de marché vont du plus scolarisé (oeuvres expérimentales) au moins scolarisé (art populaire et événements sportifs).

Par ailleurs, on doit remarquer aussi qué le potentiel d'attrait touristique varie d'un évếnement à l'autre. Ainsi, le Festival de thếâ= tre des Amériques et le Festival Juste pour Rire attirent peu de public de l'extérieur du Québec, rsepectivement $11 \%$ et $7 \%$ alors que I'Exposition Picasso et le Grand Prix Labatt en attirent beaucoup plus ( $36 \%$ et $45 \%$ ). Donc, pour un même segment de marché, des événements peuvent différer grandement quant à leur potentiel d'attraction touristique, certains joueront un rôle moteur, d'autres auront un impact plus local. On pourrait ajouter que parmi ces derniers, certains profiteront en fait du public hors Québec amené par des manifestations concurrentes; c'est le cas du Festival Juste pour Rire dont seulement $27 \%$ de la clientèle touristique déclare être venue spécifiquement pour l'événement. Ces résultats concordent avec ceux trouvés par les auteurs de cet article dans leur érude du Musée des Beaux-Arts de Montréal, du Festival International de Jazz de Montréal et de l'Orchestre symphonique de Montréal ${ }^{\text {[9] }}$.

\section{Quelques implications stratégiques}

Il convient de souligner, à la lumière des discussions précédentes, qu'un événement qui profite du pouvoir d'attraction d'une autre manifestation ne doit pas être considéré comme parasite de cette autre manifestation. En effet, le touriste peut fréquenter une région pour plusieurs raisons différentes; il ne le fait pas cependant parce qu'il veut visiter des usines. Règle générale, l'attrait d'un coin de pays vient d'une série de facteurs, la concentration d'offre culturelle, de gastronomie ou de beauté naturelle. Ceci nous mène à une première constatation d'ordre stratégique: différents événements se complètent, certains font office d'attirance vers une région, d'autres contribuent à y garder le touriste plus longtemps et certains en se conjuguant peuvent constituer un attrait colossal (ex.: Ramsès combiné à Picasso). De façon idéale done, chaque région devrait développer une offre touristique diversifiée qui tient compte de ces trois volets d'une même mission.

La deuxième constatation tient à la segmentation du marché qui peut être effectuée par le biais d'une offre culturelle. Nous avons vu que le touriste culturel possède des caractéristiques particulières; il est scolarisé donc plus sophistiqué, et l'on pourrait ajouter qu'il a de l'argent à dépenser. Pour accroitre le nombre de visiteurs dans leur région, les res- 


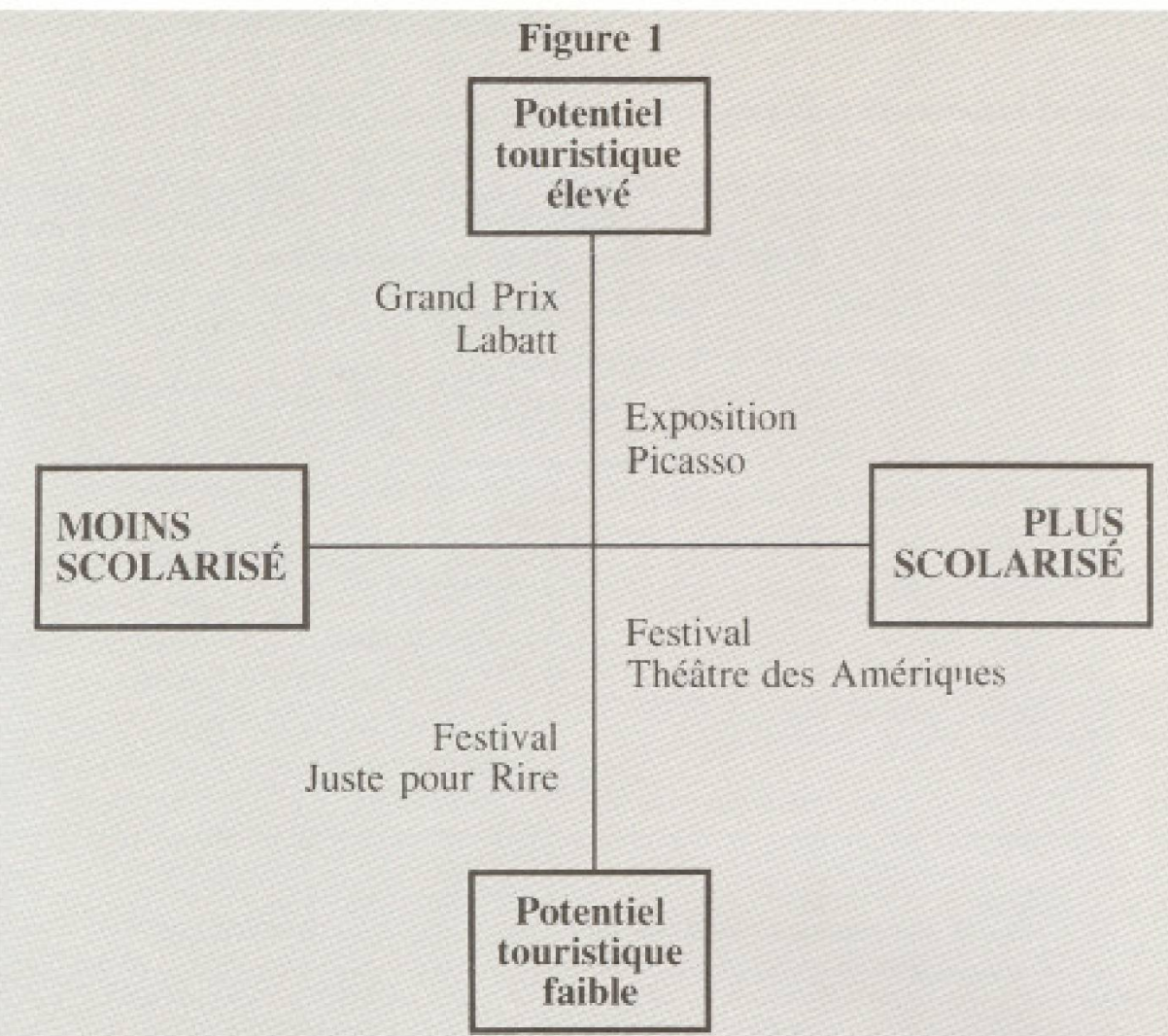

$\begin{array}{llll}\text { Événements } & \text { Arts } & \text { Oeuvres } & \text { Oeuvres } \\ \text { sportifs } & \text { populaires } & \text { classiques } & \text { expérimentales }\end{array}$

ponsables touristiques pourraient vouloir étatler sur ces types d'evenements pour attirer une catégorie de clientele non actuellement représentée parmi les visiteurs de ladite région.

Par ailleurs, toutes les régions ne présentent pas le même genre de potentiel touristique. C'est notre troisième constatation. Des régions densément peuplées comme Montréal, Québec ou Ottawă, peuvent se permettre une offre très diversifiéc où tous les types d'événements sont représentés, de l'art élitiste à l'art populaire, aux sports. A l'inverse, certaines régions doivent se spécialiser, doivent reposer sur un elément touristique fort. Si cet element est relie a la gastronomie, aux sports ou aux beautés naturelles, des organismes culturels pourront allors éprouver de la difficulté à attirer de la clientèle additionnelle si leur marché-cible n'est pas compatible avec le profil du consommateur qui visite leur région. Pour palier à ce manque à gagner, le développement de produits culturels appropriés avec une communication ciblée pourra susciter un déplacement de court sejour du touriste culturel grande région vers une rếgion moins densément peuplée.

Enfin, whe dernière constatation, une région pourrait decider de diversifier son offre touristique et de développer le volet culturel. Ce faisant, elle attaquerait un segment de marché differrent et pourrait ainsi augmenter son nombre total de visiteurs. Il faut bien sûr au préalable savoir à quel segment du marché s'adresse l'offre présente; il pourrait arriver que le profil de la clientèle d"un événement ou d'un produit touristique conventionnel corresponde à celui du touriste culturel. auquel cas on ne diversifierait pas véritablement notre offre.

On peut ajouter un corrolaire à notre quatrième constatation. Une région qui offre plusieurs types de produits touristiques aurait intérết à aussi développer des stratégies de communications commerciales de masse diversifífes. Les segnents de marché visés étant différents, il serait justifié de cibler les campagnes de publicité, de promotion des ventes et de relations publiques de façon à atteindre le plus efficacement possible les publics désirés.

Des quatre constatations stratégiques précédentes, nous pouvons degager certaines generalisations susceptibles de contribuer à une gestion efficace de l'offre culturellé régionale dans le cadre d'un marché touristique.

Habituellement, la région qui désire développer son achalandage touristique tablera sur deux dimensions primordiales, notamment le type de clientèle (nouvelle vs actuelle) et le type d'activitét** (inédite vs existante).

Comme nous pouvons le constater à la figure 2 , ces deux dimensions permettent de definir soit les objectifs régionaux (allonger les séjours vs développer de nouveaux touristes) soit de qualifier l'offre (activités inédites vs des activités existantes ailleurs et importées occasionnellement dans la région).
QUADRANT I:

(Activité inédite -

\section{clientèle nouvelle)}

Certes la position produit-marché la plus difficile à gerrer puisque nous nous adressons à une clientèle nouvelle et quasi inconnue et désirons lui offrir une activité culturelle inédite c'est-à-dire qu'il nous faut créer l'événement et que le choix du mix marketing (produit-prix-place- promotion) ne repose, ă vrai dire, sur aucune expérience passée. Le Festival de Lanaudière de par son innovation et son attrait pour une nouvelle clientele pour cette région s'avềre un bon exemple clientactivité s"adressant en majeure partie à une nouvelle clientèle touristique régionale et très originale par rapport aux activités disponibles dans les autres régions du Québee.

\section{QUADRANT II: (Activité inédite - clientèle actuelle)}

Bien qu'aussi difficile à implanter que la position produit-marché du quadrant IV, la principale difficulté réside ici dans la création et la sélection d'activitếs culturelles à offrir de manière à répondre aux besoins de notre clientèle touristique actuelle afin de prolonger le séjour et conséquemment d'accroitre les dépenses au scin de la région. Lorsque Mont-Laurier envisage d'établir un centre d'interprétation muséologique sur la chasse et la pêche sachant que sa principale clientèle touristique actuelle est composee de pêcheurs et de chasseurs, les autorités touristiques locales cherchent avant tout à accroitre à la fois la durée du séjour et les dépenses des louristes en région.

\section{QUADRANT III (Activité existante - clientèle actuelle)}

Bien que la position relative produit-marché la moins difficile à implanter puisque la clientèle est connue et la performance de l'activité est déjà vérifiée, cette position produit-marché peut s"avérer fort pertinente si l'objectif est d'allonger la durée du séjour. Deux equipes de la Ligue Nationale d'Improvisation peuvent effectuer une soirée d'impro sur l'histoire ou sur des événements historiques majeurs caractérisant lạ région de Québec.

\section{QUADRANT IV (Activité existante - clientèle nouvelle)}

Tel que souligné au quadrant II, la position produit-marché qui y prévaut revết une difficulté moyenne d'implantation, difficulté qui réside surtout dans la connaissance minimale de la clientèle nouvelle. Le cas d'un thétre d'étế programmant annuellement une piece à succès sur le marché américain peut certainement attirer une clientèle américaine qui séjourne, par exemple ă Montréal, qui a déjà entendu parler de la pièce sans y avoir assisté ct qui, si sollicitée adéquatement pourrait être intéressée à se déplacer dans la région offrant cette activité culturelle. 


\section{Conclusion}

Le consommateur culturel représente un segment du marché particulier qui peut faire en sorte d'accroitre le flot touristique d'une région. Les quelques études réalisées jusqu'à ce jour nous permettent de cerner le profil socio-démographique de cette clientèle particulière et de la mettre en relation avec les autres publics d'événements sportifs, de loisirs et autres. Notre connaissance de ce marché particulier gagnerait cependant si nous pouvions obtenir le profil du consommateur des autres types de produits touristiques.

L'analyse du profil socio-démographique des divers types de touristes nous donne l'oocasion de procéder à une première segmentation du marché. Cependant, comme nous l'avons aussi souligné, quoique la scolarité obtenue soit un bon prédicteur de la propension du citoyen à fréquenter les arts, une analyse de type psychologique complèterait encore mieux notre connaissance du sujet et contribuerait à l'amélioration du contenu des stratégies de mise en marché du produit touristique, culturel ou non. En tout état de cause, nous savons que le consommateur culturel peut représenter un potentiel touristique intéressant pour une région. Il reste à trouver les moyens à bâtir une offre intéressante et d'en faire la promotion. $f$

"Cette proportion est exprimée en termes de médiane.

"Nous soulignons ici les termes "ellitiste" et "popula. rite" dans un sens qui ne porte pas de jugement de valeur ni nogatif, ni positif.

".Par activite, nous nous situons à un niveau d'abstrac" lion au-dela-de la catégorisation des sept arts.

\section{Bibliographie}

(1) COLBERT, Francois, "Le bovis, te thistre of le thestre d"été". Jeu, Cahiers de théatre, numéro 42 . 1997, pp. 127 a 134

(2) COLBERT, Francois, "Le prochain defi de la commencialisation des produits culturels"; Questions de culture, wolume 7,1984 , pp. 127 a 138.

(3) CANADA COUNCIL, A Survey of Arts Audience Studies: A Canadian Perspective 1967-1984, septembre 1984.

(4) BOOK, S.H. et GLOBERMAN, S., The Audience for The Performing Arts, Ontario Arts Council, 1974.

(5) DEMAND RESEARCH CONSULTANTS INC., The Market for Modern Dence and The Market for Classical Dance. Ottawa, 1982.

(6) NATIONAL ENDOWMENT FOR THE ARTS. Audience Studies of The Performing Arts and Museums: A Critical Review, novembre1978.

(7) TIGHE, Anthony J., "Cultural Tourism in the U.S.A. ". Tourism Management, December 1985, p. 234 a 251

(8) ECONOSULT INC., Résultats d'un sondage auprés de la clientèle de neuf événements de "Rendezvous Montréal 1985", septembre 1985.

(9) COLBERT, F. et BOISVERT, J.-M. Étude de eertaines dimensions écenomiques des activités à caractere culturel: le cas de l'Orchestre Symphonique de Montréal, du Musée des Beaux-Arts de Montréal et du Festival International de Jazz de Montrubal, Ville de Montrél et Ministène des Affaires culturelles du Quebec, mars 1965.

Quadrant II

OBJECTIF:

Allonger le séjour et accroitre les dépenses.

\section{OFFRE:}

Des activités originales que l'on ne retrouvent pas en d'autres régions.

EX:

Centre d'interprétation muséologique de chasse et péche.

Figure 2

\section{Tourisme et activités culturelles}

\section{ACTIVITÉ INÉDITE}

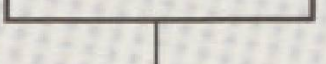

Quadrant I

OBJECTIF:

Développer nouveaux touristes.

OFFRE:

Des activites originales que I'on ne retrouvent pas en d'autres régions.

EX.:

Le Festival de Launaudière

\section{CLIENTÈLE} NOUVELLE

OBJECTIF:

Allonger le séjour et accroitre les dépenses.

\section{OFFRE:}

Des activités que l'on retrouvent dans d'autres régions.

EX:

La LNI en tournée qui s"arrête dans la région.

\section{Quadrant III}

\section{ACTIVITÉ EXISTANTE}

OFFRE:

Des activités que l'on retrouvent dans d'autres régions.

EX.:

Un théătre d'été.

\section{Quadrant IV}

